\title{
ABSTRACTS OF THESES
}

S. G. Mohanty, On some properties of compositions of an integer and their application to probability theory and statistics. University of Alberta, April 1961 (Supervisor, T.V. Narayana).

The purpose of this thesis is to investigate combinatorial properties of partial orders defined on compositions of an integer, and to apply these properties to yield a unified approach to various problems in probability and statistics. The type of problems we deal with in probability are described as "ballot problems" by Feller. We show that ballot problems can most conveniently be treated by the study of partial orders defined on compositions of an integer. As an application of the same ideas to problems in statistics, we consider several equivalent characterizations of simple sampling plans of size $\mathrm{n}$ and show that structurally, the problem of characterizing simple sampling plans is formally identical to the study of partial orders on compositions of an integer.

In Chapter I a résumé of the relevant work on the compositions of an integer is presented. We extend and continue this work, bringing out more clearly its relation to ballot problems. Two simple and new : methods are discussed, enabling us to rederive and extend most of the known results in this field.

In Chapter II, we prove that simple sampling plans of size n can be characterized by their boundary points. By the consistent use of the partial orders mentioned above, we obtain 1:1 correspondence between simple sampling plans of size $\mathrm{n}$ and simple symmetric sampling plans of size $2 \mathrm{n}$. Certain topics and theorems which have been touched upon but not fully discussed in the literature are brought out as a natural consequence of our methods.

In Chapter III, application of the same concept has been made to other problems in probability theory and statistics. A brief mention of number-theoretic results which follow as a by-product of our approach concludes the chapter.

George C. Bush, On embedding a semigroup in a group. Queen's University, April 1961 (Supervisor, I. Halperin).

A given semigroup $A$ is said to be embeddable in a group if there 
exists a group $G$ which contains a subsemigroup isomorphic to $A$. If $A$ is commutative or if each pair of elements of $A$ has a common left multiple, there always exists a group $G$ such that such an embedding of $A$ in $G$ is possible. In the general case a semigroup may or may not be embeddable in a group.

Different recessary and sufficient conditions for the embeddability of a semigroup in a group were given first by Malcev (1939) and by Lambek (1951).

Malcev introduced formal left and right inverses for elements of $A$ and constructed a group $G$ from the equivalence classes of words formed from elements of $A$ and formal inverses. He then derived a set of conditions necessary and sufficient that $A$ be isomorphic to a subsemigroup of this $G$. Each Malcev condition is associated with a chain of transformations on the se words. The thesis gives an exposition of this work of Malcev, but uses simpler proofs due to Tamari.

Lambek considered ordered pairs of elements of A that have a common left multiple and called every such pair a ratio. In the set of ratios he defined a partial multiplication. Equivalence classes of finite sequences of ratios a re used to form a group. A necessary and sufficient condition that the semigroup be embeddable is that all the associative laws hold for the partial multiplication of the ratios. Lambek formulated conditions on the semigroup for this to be true. He gave the se conditions in terms of Eulerian polyhedra and used geometric properties in his proof. The the sis represents each associative law by a $2 n$-tuple and with each 2 n-tuple associates a Lambek condition. In this way a sufficient subset of the Lambek conditions is obtained without using geometric considerations.

The Lambek conditions associated with polyhedra with exactly two vertices were named the "Iunar" conditions by Halperin. Jackson studied these conditions, but did not answer the question of the sufficiency. The thesis considers these lunar conditions and a related set called the dual Iunar conditions, and establishes that the two sets taken together are not sufficient for the embeddability of a semigroup in a group.

That the individual Malcev and the individual Lambek conditions are not identical is shown by two counter-examples. It is also shown that the only conditions that have the form of a Malcev condition and also that of a Lambek condition are the lunar conditions.

One condition is said to be a consequence of anothe $r$ if the first is satisfied by every semigroup that satisfies the second. Proofs are given that each Lambek condition is a consequence of a single Malcev condition and that each Malcev condition is a consequence of a single Lambek condition. 
Malcev proved that no finite set of conditions of a certain form. (which includes the Malcev and Lambek conditions) could be necessary and sufficient for the embeddability of a semigroup in a group, but his proof requires some clarification and correction. This is given in the thesis.

W. Bruce Antliff, The limit of ${ }^{(n)} m\left(E_{(n)} x\right)$ as $n \rightarrow \infty$, and conditions which are equivalent to compressibility. Queen's. University, April 1961 (Supervisor, I. Halperin).

As the title of the the sis suggests, it deals with-two separate topics. The first concerns itself with the Cartesian product $(\mathrm{X}, \mathrm{S}, \mathrm{m})$ of a denumerable number of measure spaces. Such a product can also be considered as the Cartesian product of two spaces $X={ }_{(n)} X \times(n) X$, with the associated measurable sets $S={ }_{(n)} S \times{ }^{(n)} S$ and measures $m=(n)^{m} \times{ }^{(n)} m$, where $\left((n) X,(n)^{S,}(n)^{m}\right)$ is the Cartesian product of the first $n$ components of $(X, S, m)$ and $\left({ }^{(n)} X,{ }^{(n)} S,{ }^{(n)} m\right)$ is the Cartesian product of the remaining components of $(\mathrm{X}, \mathrm{S}, \mathrm{m})$. It is proved, in the first section of this thesis, that, for every measurable set $E$ in $X$, there exists a null subset $N$ such that if $E^{\prime}=E-N$ then $\lim _{n \rightarrow \infty}{ }^{(n)} m\left(E^{\prime}(n)^{x}\right)=1$ for every $\left.x=(n)^{x},{ }^{(n)} x\right) \in E^{\prime}$.

The second section of the the sis deals with transformations of a space into itself.

A transformation is called measurable if $T^{-1} E$ is measurable for every measurable set $E$ and a transformation will be called inversely measurable if $T E$ is measurable for every measurabie set $E$.

A set $E$ is said to be $k$-corresponding to a set $F$, if the re exists a decomposition $E=U_{i=1}^{k} E_{i}$ with the $E_{i} s$ disjoint and measurable and a decomposition $F=U_{i=1}^{k} F_{i}$ with the $F_{i}$ s disjoint and measurable such that for each $i, T^{n_{i}} E_{i}=F_{i}$ where $n_{i}$ represents an integer (possibly negative.

It had been proved by P. R. Halmos in 1946 that, if $T$ is a oneto-one transformation which is inversely measurable, then the following three conditions are equivalent to each other.

(1) $T$ is compressible.

(2) For some positive intege $r$ and some measurable set $E, E$ is $k$-corresponding to a proper subset of itself. 
(3) For some positive integer $\mathrm{k}, \mathrm{X}$ is $\mathrm{k}$-corresponding to a proper sub set.

In conditions (2) and (3) the term proper subset means a subset which differs from the original set by more than a null set.

The principal part of this section of the thesis consists of a theorem in which the three conditions mentioned in the preceding paragraph are proved to be equivalent, but the proof does not require the transformation to be one-to-one and it is valid for either a measurable transformation (provided that $\mathrm{TX}$ is measurable) or an inversely measurable transformation.

An application of this theorem is then made to strong recurrence. An element $x$ of a set $E$ is said to be recurrent if $T^{n} x \in E$ for at least one value of $n$, and $x$ is said to be strongly recurrent if $T^{n} x \in E$ for an infinite number of values of $n$. A transformation is said to be conservative if every set of positive measure contains at least one point which is recurrent under $T$. The theorem mentioned above is applied to prove that, if $T$ is a conservative and measurable transformation and if $\mathrm{TX}$ is measurable. then almost all points of any measurable set are strongly recurrent. 